OPEN ACCESS

Edited by: Rik Vandenberghe, KU Leuven, Belgium

Reviewed by:

Chiao-Yi Wu

Nanyang Technological University,

Singapore

Tom Theys,

University Hospitals Leuven, Belgium

${ }^{*}$ Correspondence:

Ruijie Wu

wuruijie8381@foxmail.com

Received: 12 January 2019

Accepted: 20 May 2019

Published: 05 June 2019

Citation:

Ni B, Wang $X, Y u T$, Wu R and

Wang $B$ (2019) Pre-surgical

Language Mapping in Epilepsy: Using

fMRI in Chinese-Speaking Patients.

Front. Hum. Neurosci. 13:183.

doi: 10.3389/fnhum.2019.00183

\section{Pre-surgical Language Mapping in Epilepsy: Using fMRI in Chinese-Speaking Patients}

\author{
Bing $\mathrm{Ni}^{1}$, Xueyuan Wang ${ }^{1}$, Tao Yu ${ }^{1}$, Ruijie $\mathrm{Wu}^{2,3 *}$ and Bo Wang ${ }^{2,3}$ \\ ${ }^{1}$ Department of Functional Neurosurgery, Xuanwu Hospital, Capital Medical University, Beijing, China, ${ }^{2}$ State Key Laboratory \\ of Brain and Cognitive Science, Institute of Biophysics, Chinese Academy of Sciences, Beijing, China, ${ }^{3}$ Beijing MR Center \\ for Brain Research, Beijing, China
}

Accurate localization of language processing areas is critical in patients undergoing epilepsy surgery. In this study, we aimed to use functional magnetic resonance imaging (fMRI), which is a non-invasive mapping method, to establish a panel of tasks investigating patients' language function. We developed six tasks, including a series of progressive comprehension tasks from words, sentence to text, a verb generation task that can detect subtle left-brain activation, an auditory comprehension task that explored the temporal language-related areas, and a visual object-naming task provided for poorly educated patients. We successfully located the language cortex in 40 patients, and subsequently determined hemispheric dominance for the Chinese language. Our results showed a concordance between fMRI tasks and electrical cortical stimulation. The consistency across tasks revealed by the laterality index, as well as the concordance between the surgical outcomes and the results of localization, suggested the validity of our fMRI tasks. Our fMRI tasks also corroborate and extend the finding that the left middle frontal area (BA 9) plays an important role in reading Chinese.

Keywords: electrocortical stimulation mapping, epilepsy, Chinese language, functional magnetic resonance imaging, language processing

\section{INTRODUCTION}

Several approaches have been used to clinically identify the specific cortices and/or dominant hemisphere associated with language, including electrocortical stimulation mapping (ESM) through intracranial electrodes (Ojemann et al., 1989), transcranial magnetic stimulation (Epstein, 1998), the intracarotid sodium amobarbital perfusion test (Wada test) (Strauss and Wada, 1983; Baxendale, 2009), and functional neuroimaging techniques (Bookheimer, 2002).

The Chinese language differs from alphabetical languages such as English in many aspects, including orthography, phonology, and syntax (Kochunov et al., 2003; Qiu et al., 2006). Mandarin has an ideographic script, requiring one to memorize the phonology and meaning of each character to vocalize and comprehend. Thus, brain activation in processing the Chinese language is expected to differ from that in processing English. Valaki et al. (2004) suggested that there was greater symmetry in brain hemispheric dominance in Mandarin speakers evaluated by magnetoencephalography (MEG), whereas asymmetry was obvious in English and Spanish speakers (Valaki et al., 2004). Among those study participants, 100\% of the Spanish-speaking 
population, $80 \%$ of the English-speaking population, and only $14 \%$ of the Mandarin-speaking population were lefthemisphere dominant.

The purposes of the pre-surgical evaluation for epilepsy include establishing the location and extent of the seizure focus and its relationship to brain function, such as localization of language and other realms of cognitive function. These findings can assist the neurosurgical planning team in determining proper surgical boundaries and preserving vital functions.

In patients with epilepsy, it is especially important to delineate language areas prior to surgery (Duffau et al., 2003; Hamberger, 2007; Cummine et al., 2009), as functional anatomy may be reorganized with a transfer of functions to other areas in the ipsilateral or contralateral hemisphere. For example, functional imaging studies of language processing in patients with chronic epilepsy with a left hemisphere focus have provided evidence for a preoperative right hemispheric activation shift (Billingsley et al., 2001; Gaillard et al., 2002; Rutten et al., 2002; Sabsevitz et al., 2003; Carpentier et al., 2010). Surgical resection of epileptogenic regions requires knowledge about any shift of language function, as it may serve as a prognostic indicator and/or impact the surgical approach, with the potential to minimize neurologic deficits post-surgery.

The ESM is a golden standard for the language mapping. It can directly test any interruption during the stimulation on the language related brain areas. However, for some patients the language task cannot produce any positive result during the stimulation. For the patients whose lesion was on the right hemisphere, the left language related areas could n't be stimulated due to clinical reason. As a result, fMRI as a non-invasive approach could be an alternative method for those who cannot be tested with ESM.

In our study, we aim to develop a series of fMRI tasks to locate Chinese language related areas before an epileptic surgery. Such tasks should reliably activated Broca's and Wernicke's areas on individual level in a relatively short scanning time. We compared our fMRI results with ESM results to verify the validity of our fMRI tasks. Also, we compared the results across different fMRI tasks and calculated the lateralization index to test the reliability of our fMRI tasks.

\section{MATERIALS AND METHODS}

\section{Participants}

Forty patients (24 male, 16 female) ranging from 9 to 40 years old (mean \pm SD: $25.05 \pm 9.05$ years) were enrolled in the study after giving informed consent. Thirty-six patients were right-handed and four were left-handed native Chinese (Mandarin) speakers from the mainland of China. They all underwent preoperative fMRI between the years 2015 and 2018. In addition, they all met the following inclusion criteria: (1) diagnosed with refractory symptomatic partial epilepsy and sought resective surgery between 2015 and 2018 at the Beijing Institute of Functional Neurosurgery; (2) subjected to noninvasive evaluation of epileptogenic zones in the dominant hemisphere and those potentially close to, or overlapping language areas; and (3) showed signs of normal intelligence and language function preoperatively. The history of epilepsy in these patients ranged from 3 to 38 years (mean \pm SD: $13.20 \pm 7.47$ years). In Table $\mathbf{1}$, we present whether the resection areas involved Broca's area (inferior frontal and middle frontal gyri, including Brodmann areas 44, 45, 9, and 46) and Wernicke's area (supramarginal, angular, and superior temporal gyri, including Brodmann areas 22, 21, 39, and 40). None of the patients' resection areas included the language cortex defined by our tasks. After surgery, language function in the patients was tested using the Chinese Language Aphasia Test in the immediate postoperative period ( 3 days post-surgery). The study was conducted in accordance with the ethical principles of the 1964 Declaration of Helsinki. The Institutional Review Boards of the Xuanwu Hospital approved all procedures.

\section{Electrocortical Stimulation Mapping (ESM)}

Twenty patients underwent craniotomy for implantation of subdural electrodes or stereotactic electroencephalography (sEEG) for localization of epileptogenic foci. Custom subdural grid arrays or sEEG were designed for each patient based on seizure semiology, ictal and interictal scalp video-EEG recordings, and structural MRI.

Intraoperative ESM was performed in patients during the craniotomy. Electrical stimulations were delivered according to the following parameters: bipolar, biphasic, frequency $33 \mathrm{~Hz}$, and pulse duration $0.2 \mathrm{~ms}$, intensity $0.1-18 \mathrm{~mA}$, stimulation duration 3 s. Extraoperative ESM was performed in patients before the craniotomy. Electrical stimulations were delivered according to the following parameters: bipolar, biphasic, frequency $50 \mathrm{~Hz}$, and pulse duration $0.2 \mathrm{~ms}$, intensity $0.1-6 \mathrm{~mA}$, stimulation duration 3 s. The stimulation of language areas was delivered at the threshold previously identified to produce a motor response, which was specific for each patient. During ESM, the intracranial EEG was monitored for after discharges and seizures.

Stimulation was applied to electrodes overlying the areas defined by our fMRI tasks in frontal and temporal lobe language areas. During stimulation, the patients were asked to perform a counting task and a question-and-answer task. If there was a change in language performance during stimulation of a specific electrode, stimulation was subsequently repeated during the testing session to ensure reliability. An anatomical region of the brain was determined as necessary for language function, if errors were observed on testing and repeat testing, and no after discharges or seizures were evident on EEG to account for those errors.

\section{Language Tasks}

Prior to fMRI scanning, tasks were explained to all patients, who were trained in their performance using stimuli different from those presented during the scan, to ensure adequate understanding and performance. In the scanner, all tasks were performed covertly to minimize head motion.

The age and educational background of all 40 patients varied considerably, which led to considerable differences in their ability 
TABLE 1 | Descriptive characteristics.

\begin{tabular}{|c|c|c|c|c|c|c|}
\hline Patients no. & Handedness & Lesion & Surgical resection & $\begin{array}{l}\text { Whether BA } \\
9 / 44 / 45 / 46 \text { was } \\
\text { involved in resection }\end{array}$ & $\begin{array}{l}\text { Whether BA } \\
21 / 22 / 39 / 40 \text { was } \\
\text { involved in resection }\end{array}$ & $\begin{array}{l}\text { Post-surgery language } \\
\text { function }\end{array}$ \\
\hline 1 & $\mathrm{R}$ & EpiF on LT & LT, LH & No & LBA21, 22 & Intact \\
\hline 2 & $\mathrm{R}$ & LFTP EF & LT, LH, WM D & LBA9, 44, 45, 46 & L BA21, 22, 39, 40 & Intact \\
\hline 3 & $\mathrm{R}$ & EpiF on LHT & J of LT, LO, LP & No & L BA39 & Intact \\
\hline 4 & $\mathrm{R}$ & tumor on LT & $\mathrm{LT}$ & No & LBA 21, 22 & Less fluency \\
\hline 5 & $\mathrm{R}$ & LF SP & LF & LBA44, 45, 46 & No & Intact \\
\hline 6 & $\mathrm{R}$ & space occupying on L LaV & LT, L LaV & No & LBA21, 22 & Intact \\
\hline 7 & $\mathrm{R}$ & LFTP A & $\mathrm{LT}$ & No & LBA21, 22 & Intact \\
\hline 8 & $\mathrm{R}$ & EpiF on LTO & LT, LH & No & LBA21, 22 & Intact \\
\hline 9 & $\mathrm{R}$ & LHemi FCD & F, T, O WM Fiber D & LBA9, 44, 45, 46 & LBA21, 22, 39, 40 & Intact \\
\hline 10 & $\mathrm{R}$ & LHemi V, HGM, FCD & $\mathrm{LT}$ & No & LBA21, 22 & Intact \\
\hline 11 & $\mathrm{R}$ & LHemi A & F, T, O WM Fiber D & LBA9, 44, 45, 46 & L BA21, 22, 39, 40 & Intact \\
\hline 12 & $\mathrm{R}$ & LFIT FCD & LF, LT, LI, LP & LBA9, 44, 45, 46 & L BA21, 22, 39, 40 & Intact \\
\hline 13 & $\mathrm{R}$ & EpiF on LF & $\mathrm{LT}$ & No & L BA21, 22 & Intact \\
\hline 14 & $\mathrm{R}$ & $\mathrm{AO}$ on $\mathrm{RF}$ & $\mathrm{RT}$ & $\mathrm{R} B A 45,46$ & R BA21, 22 & Intact \\
\hline 15 & $\mathrm{R}$ & LHemi A, EF & $\mathrm{LT}$ & No & LBA21, 22 & Intact \\
\hline 16 & $\mathrm{R}$ & Cerebellar atrophy & $\mathrm{RT}, \mathrm{RH}, \mathrm{Rl}$ & No & R BA21, 22 & Intact \\
\hline 17 & $\mathrm{R}$ & LHemi FCD, LPO EF & $\mathrm{J}$ of LP, LO, LT & No & LBA21, 22, 39, 40 & Intact \\
\hline 18 & $\mathrm{R}$ & EpiF on LOT & $\mathrm{J}$ of LT, LO, TWM D & No & LBA21, 22, 39, 40 & Intact \\
\hline 19 & $\mathrm{R}$ & No & LT & No & L BA21, 22 & Intact \\
\hline 20 & $\mathrm{R}$ & EpiF on LPF & LP, LF & L BA45, 46 & LBA39, 40 & Intact \\
\hline 21 & $\mathrm{R}$ & EpiF on LT & $\mathrm{LT}$ & No & LBA21, 22, 39, 40 & Intact \\
\hline 22 & $L$ & LHemi A, LTP EF & LC, LT, LP, O WM D & No & L BA21, 22, 39, 40 & Intact \\
\hline 23 & $\mathrm{R}$ & EpiF on LO, FCD & J of LT, LO & No & LBA21, 22 & Intact \\
\hline 24 & $\mathrm{R}$ & LFTP EF & $\mathrm{LT}$ & No & LBA21, 22, 39, 40 & Intact \\
\hline 25 & $L$ & $\mathrm{~J}$ of LFTP EF & LT, LP & No & LBA39, 40 & Less fluency \\
\hline 26 & $\mathrm{R}$ & LFTP FCD & LT, LH, LF, LP,WM D & LBA9, 44, 45, 46 & LBA21, 22, 39, 40 & Intact \\
\hline 27 & $\mathrm{R}$ & EpiF on LHT & LT, LP & No & L BA39, 40 & Intact \\
\hline 28 & $\mathrm{R}$ & EpiF on LFT & LF, LP & L BA45, 46 & No & Intact \\
\hline 29 & $\mathrm{R}$ & EpiF on LF & LF, LI & L BA45, 46 & No & Intact \\
\hline 30 & $\mathrm{R}$ & AO on BF, LF HGM & LF & LBA9, 44, 45, 46 & No & Less voluntary speaking \\
\hline 31 & $L$ & LFTPO EF & Lf, LP & LBA9, 44, 45, 46 & L BA39, 40 & Intact \\
\hline 32 & $\mathrm{R}$ & LF FCD & LC, LF & L BA45, 46 & No & Less fluency \\
\hline 33 & $\mathrm{R}$ & EpiF on LT & $\mathrm{LT}, \mathrm{LP}$ & No & LBA21, 22, 39, 40 & Intact \\
\hline 34 & $L$ & LHA & LF & LBA9, 44 & No & Intact \\
\hline 35 & $\mathrm{R}$ & LLaV & LF & LBA9, 44, 45, 46 & No & Intact \\
\hline 36 & $\mathrm{R}$ & LHA & LP, LT, LH & No & LBA21, 22, 39, 40 & Intact \\
\hline 37 & $\mathrm{R}$ & EpiF on LT & $\mathrm{LT}$ & No & LBA21, 22 & Intact \\
\hline 38 & $\mathrm{R}$ & EpiF on LC & LC, LT, LP & No & L BA39, 40 & Intact \\
\hline 39 & $\mathrm{R}$ & EpiF on LHFT & LF, LT, LP & LBA44, 45, 46 & L BA40 & Intact \\
\hline 40 & $\mathrm{R}$ & EpiF on LP & LP & No & No & Intact \\
\hline
\end{tabular}

F, frontal; T, temporal; C, Central; P, parietal; O, occipital; R, right; L, left; LaV, lateral ventricle; Hemi, hemisphere; J, junction; WM, white matter; D, disconnection; EF, encephalomalacia focus; EpiF, epileptogenic focus; FCD, focal cortical dysplasia; AO, artery occlusion; HGM, heterotopic gray matter; A, atrophy; SP, schizencephaly; V, ventriculomegaly.

to complete the language comprehension tasks. We designed three tasks to explore the Broca's area (inferior frontal and middle frontal gyri, including Brodmann areas 44, 45, 9, and 46).

\section{Text Reading}

We applied two different texts with about 300 Chinese characters separately in two trials. Each text was shown for $30 \mathrm{~s}$, during which the patient was required to silently read the text and understand its meaning. Three interleaved control trials, each lasting $20 \mathrm{~s}$, were conducted, which entailed the use of a blank screen with only a fixation cross.

\section{Sentence Reading}

We used a sentence-reading task to test semantic decision making, which robustly activates language-related brain areas, particularly regions contributing to speech perception and 
lexical-semantic processes. Two test trials (30 s each) were interleaved with three control trials $(20 \mathrm{~s}$ each). In the test trials, we presented eight simple sentences with a question regarding common sense in daily life that could have been answered with a brief "yes" or "no" (e.g., "Is your name Zhang Xiaohong?"). The patients were required to silently read every sentence and answer each question. All eight sentences were shown on the screen, and the patients were able to adjust their speed of reading. The control trials entailed the use of a blank screen with only a fixation cross.

\section{Word Comprehension}

We employed a word interpretation task to further investigate activation in Broca's area. The trial structure was the same as that in the two previous tasks. In the test trials, 16 two- or three-character nouns were shown on a screen. The patients were required to silently read the words and determine whether the object described by the word was living or inanimate. The words in the two test trials were different from each other. In the control trials, a blank screen was shown with a green fixation cross.

Altogether, the three tasks provided a set of complementary tests that could be adapted to various educational backgrounds. Any overlap in the activation areas associated with the three tasks could provide more convincing results regarding language comprehension. In addition to the previous tasks, we also employed a verb generation task, a visual object naming task, and an auditory comprehension task to evaluate more language processes.

\section{Verb Generation}

Verb generation shows strong left language lateralization indices (LI) in right-handed healthy controls (Petersen et al., 1988). These LIs are consistent with results of more invasive methods, such as Wada testing and intraoperative stimulation in patient populations. In one trial, the patients were instructed to sequentially read 16 nouns, and silently generate a semantically related verb, one after the other (e.g., "dumpling - eat"). Patients were asked to adjust their individual speed of reading. Two 30-s test trials were interleaved with three 20-s control trials. A blank screen with a fixation cross was presented in the control trials.

\section{Visual Object Naming}

Naming is considered a left-hemisphere function that operates according to a posterior-anterior specificity gradient, with more fine-grained information being processed in the most anterior regions of the temporal lobe (ATL), including the temporal pole (TP). Difficulties in word finding are typically assessed using visual confrontation naming tasks, and have been associated with selective damage to the ATL resulting from various etiologies (Campo et al., 2016). We applied 15 stick figures representing 15 daily objects in each of two test trials. The patients were required to silently name each of the objects in sequence. Each of the two test trials lasted $30 \mathrm{~s}$, which were interleaved with three 20-s control trials. A blank screen with a fixation cross was presented during the control trials.

\section{Auditory Comprehension}

The auditory comprehension task comprised the auditory version of a word interpretation test. It robustly activated languagerelated brain areas, particularly those regions contributing to speech perception and lexical-semantic processes. The degree of lateralization of these activated areas corresponds with Wada language asymmetry in individual epilepsy patients. We applied this task to investigate the language processing areas related to Wernicke's area. The trial structure was the same as those in the previous tasks. In the test trials, the patients passively heard about 10 to 16 words, read in Mandarin, describing daily objects. During a brief pause between the words, the patients were required to silently decide whether the object just referred to was living or inanimate. The number of words heard and length of the brief pause between words varied among patients, based on differences in the educational background of each patient. In the three 20 -s control trials, patients only heard the noise of the scanner.

\section{fMRI Data Acquisition}

All imaging was conducted using a three Tesla Siemens Medical systems Prisma scanner at the Beijing Brain MRI Center. Whole brain anatomical scans were acquired using a high resolution magnetization-prepared rapid acquisition gradient echo sequence, consisting of 176 T1-weighted echo-planar image (EPI) slices of 1-mm thickness, with an in-plane resolution of $1 \times 1 \mathrm{~mm}$ [field of view $256 \mathrm{~mm}$, repetition time $(\mathrm{TR})=2530 \mathrm{~ms}$, echo time $(\mathrm{TE})=3.37 \mathrm{~ms}]$. For each of the functional tasks, T2weighted single shot gradient echo EPI scans were acquired using an interleaved ascending sequence, consisting of 60 volumes of 25 axial slices of 4 -mm thickness with an in-plane resolution of $3.4 \times 3.4 \mathrm{~mm}$ (field of view $=220 \mathrm{~mm}, \mathrm{TR}=2000 \mathrm{~ms}, \mathrm{TE}=30 \mathrm{~ms}$, flip angle $=90^{\circ}$ ). A 20 -channel Siemens head coil was used.

Informed consent complying with local institutional review board regulations was obtained from all patients. An MRI compatible light-emitting diode (LED) projector system was used to show the visual stimulus. The fMRI tasks were all block designed. During each task, a set of two trials (30 s each) was performed and interleaved with three control conditions $(20 \mathrm{~s}$ each), in which the patient was instructed to maintain fixation.

\section{fMRI Data Analysis}

All preprocessing and statistical analyses for functional images were performed using SPM12 (Statistical Parameter Mapping 12; Wellcome Department of Imaging, London) and the xjView toolbox through MATLAB 8.4 (MathWorks, Inc.). Anatomical and functional scans were reoriented to the anterior commissure, following which the functional images were co-registered to the anatomical scan. Motion correction was implemented using realignment and unwarping. The anatomical scans were segmented into gray matter, white matter, and cerebral spinal fluid, and then normalized to Montreal Neurological Institute (MNI) space. Functional images were transformed into MNI space by using the same normalization parameters. Spatial smoothing was performed using an 8 -mm full width at half maximum (FWHM) smoothing function. 
For each patient, we modeled fMRI data using the general linear model (spm12). At the individual level, statistical parametrical maps showing brain activation of each language task minus fixation were calculated at the threshold of $p<0.001$ uncorrected (at most). The usage of an uncorrected statistical threshold is justified because of the clinical reason that we were foremost interested in brain activations at the individual level. Moreover, we believe that the statistical threshold level we chose provides a balance between the risks of type-I versus type-II errors in the statistical analysis of fMRI data, especially when the focus of our test is to investigate brain activity for individual patient. Individual patient's $p$-value adjustment for each task of activation in language related areas was shown in Supplementary Table S1.

\section{Lateralization Indices (LI)}

Language cortex reorganization was studied in the Broca's area (inferior frontal and middle frontal gyri, including Brodmann areas 44, 45, 9, and 46) and Wernicke's area (supramarginal, angular, and superior temporal gyri, including Brodmann areas $22,21,39$, and 40 ), using LI to quantify the degree of lateralization of the blood-oxygen-level dependent (BOLD) signal (Rutten et al., 2002; Deng et al., 2015). It was calculated for Broca's and Wernicke's areas separately, using the following formula: $\mathrm{LI}=(\mathrm{VL}-\mathrm{VR}) /(\mathrm{VL}+\mathrm{VR})$, where VL denotes the number of voxels activated in the left hemisphere and VR denotes the number of voxels activated in the right hemisphere. In Supplementary Table S2, we provided the LIs that were calculated according to Matsuo's AveLI approach (Matsuo et al., 2012). This result was consistent with previous LIs. The LI ranged from -1 to +1 , and language lateralization was categorized into three patterns. An LI less than or equal to -0.2 was considered right-sided lateralization, whereas an LI greater than or equal to 0.2 was regarded as left-sided lateralization, and an LI between -0.2 and 0.2 suggested no clear hemispheric preference (Szaflarski et al., 2002; Backes et al., 2005).

\section{RESULTS}

In the present study, we employed comprehension tasks of text, sentences, visual words, and auditory words for all 40 patients. Moreover, in 29 patients, we added a verb generation task to detect left language lateralization. Among those patients, 20 were given a visual object-naming task in cases of relatively low educational levels. This panel of progressive tasks provided us sufficient information to determine languagerelated activations in both Broca's and Wernicke's areas, as different patients showed different levels of sensitivity to each task. One might elicit weak activation in one task but show strong activation in another. Thus, the overlapping areas of language-related activation in different tasks revealed a more reliable evidence of patients' language processing areas. Across tasks, we calculated the LI of either overlapping areas in Broca's area (including Brodmann areas 44, 45, 9, and 46) or overlapping areas in Wernicke's area (including Brodmann areas 21, 22, 39, and 40). In Table 2, the LI values of the overlapping areas and individual tasks of all patients are presented separately. The lateralization of language processing in each patient was determined according to the majority of LIs within the same indication range. In total, 17 patients showed left-sided lateralization, 18 showed right-sided lateralization, and five showed bilateral processing. When we reported the results of individual patients to the surgeons, we did not emphasize the lateralization of language processing. We aimed to comprehensively demonstrate all brain activations during language processing, especially those adjacent to the ictal focus. Here, we used the concordance between the fMRI and the ESM, LI and its consistency across tasks to measure the validity of the design of our tasks.

\section{Concordance Between fMRI and ESM}

For the 20 patients who underwent ESM, we analyzed both the ESM results and lateralization defined by our fMRI task. Concordance between fMRI and ESM was rated "high" if fMRI identified language in positively mapped sites using ESM, or if fMRI lateralized language to the opposite side of negatively mapped sites (Rodin et al., 2013). Concordance between fMRI and ESM was rated "moderate" if language was found to be lateralized on the right, with some remaining residual activations on the left hemisphere using fMRI, and ESM found positively mapped sites on the left. Concordance between fMRI and ESM was rated "low" if the fMRI failed to detect any activation on the left frontal or temporal lobes, and lateralization was determined to be on the right, and ESM was positive on the left temporal lobe. Results were labeled discordant if fMRI and ESM lateralized language to opposite hemispheres.

Our results (Table 3 ) showed that $80 \%$ of the patients showed high concordance when the fMRI and ESM were compared. Particularly when fMRI lateralized the language function on the left, ESM was able to positively identify mapping on the same side. Moderate concordance was evident in 15\% of the patients when comparisons were drawn between the two methods. In these patients, the LIs showed that language lateralization was right-sided, whereas the left hemisphere still showed some activation on the language-related areas, which were identified by ESM. The regions on the right hemisphere were not tested with electrical stimulation, since it was not exposed during the surgery or implanted with electrodes. The anatomical regions identified by ESM and fMRI were not resected during surgery, and post-surgical language function was intact. This suggests that the fMRI findings of left-brain activation were consistent with those of ESM. However, this cannot support our lateralization results on these patients.

Only one patient (5\%) showed low concordance between the two methods. The fMRI tasks failed to detect any activation on the left temporal lobe of this patient, whereas ESM produced a positive result on the left temporal lobe. Lateralization, as determined by fMRI tasks, suggested a right-sided language function. However, the post-surgical language function was intact, and part of the temporal lobe was positively mapped by ESM. The reason for the low concordance of this patient 
TABLE 2 | Language lateralization index.

\begin{tabular}{|c|c|c|c|c|c|c|c|c|c|}
\hline \multirow{2}{*}{$\begin{array}{l}\text { Patients } \\
\text { no. }\end{array}$} & \multirow{2}{*}{$\begin{array}{l}\text { Language } \\
\text { lateralization }\end{array}$} & \multicolumn{8}{|c|}{ LI } \\
\hline & & $\begin{array}{l}\text { Overlap in } \\
\text { Broca's }\end{array}$ & $\begin{array}{l}\text { Overlap in } \\
\text { Wernicke }\end{array}$ & Text & Sentence & Word & Auditory & $\begin{array}{c}\text { Verb } \\
\text { generation }\end{array}$ & $\begin{array}{c}\text { Visual object } \\
\text { naming }\end{array}$ \\
\hline 1 & bilateral & -0.17 & 0.75 & -0.37 & 0.50 & 0.03 & 0.17 & No & No \\
\hline 2 & Right & -0.99 & -0.90 & -1 & -1 & -1 & -0.79 & No & No \\
\hline 3 & Left & 0.46 & None & 1 & 0.85 & 0.33 & 0.10 & No & No \\
\hline 4 & Left & 0.61 & -0.28 & -0.08 & 0.83 & 0.64 & 0.59 & No & No \\
\hline 5 & Right & -1 & -0.58 & -1 & 0.02 & -0.66 & 0.13 & No & No \\
\hline 6 & Left & 0.02 & 1 & 0.41 & 0.63 & -0.35 & -0.04 & No & No \\
\hline 7 & Right & -0.51 & None & -0.22 & -0.11 & -0.42 & -0.95 & No & No \\
\hline 8 & Right & -0.41 & None & -0.35 & 0.48 & -0.48 & -1 & No & No \\
\hline 9 & Right & -1 & -1 & -1 & -0.20 & -1 & None & No & No \\
\hline 10 & right & -0.65 & None & None & -1 & -0.94 & None & No & No \\
\hline 11 & right & -0.98 & -1 & -1 & -0.96 & -0.28 & -1 & No & No \\
\hline 12 & right & -0.38 & None & -0.80 & -0.67 & None & 1 & -0.68 & No \\
\hline 13 & bilateral & 0.07 & 0.01 & -0.54 & 0.09 & None & -0.39 & 0.16 & No \\
\hline 14 & left & 0.01 & 0.71 & 0.80 & None & 0.71 & 0.12 & -1 & No \\
\hline 15 & right & -1 & -0.49 & -1 & -1 & None & -0.43 & -1 & No \\
\hline 16 & left & 0.65 & 1 & 0.96 & 0.58 & 0.32 & -0.14 & 0.65 & No \\
\hline 17 & right & -0.58 & -0.29 & -0.43 & None & -0.98 & -0.10 & -0.79 & No \\
\hline 18 & right & -0.62 & -0.59 & -0.96 & -0.50 & -0.66 & -0.40 & -0.62 & No \\
\hline 19 & left & 1 & 0.43 & None & 1 & 1 & 0.33 & 1 & No \\
\hline 20 & right & -0.84 & -1 & -1 & -0.58 & -0.46 & -0.02 & -0.61 & No \\
\hline 21 & right & -0.62 & None & 1 & -0.5 & -0.45 & -0.12 & 0.19 & -1 \\
\hline 22 & right & -0.45 & -0.84 & -1 & -1 & None & -1 & -0.54 & -1 \\
\hline 23 & left & 0.38 & None & 0.83 & 1 & 0.22 & 0.68 & 1 & 0.74 \\
\hline 24 & right & -0.55 & -1 & -1 & -1 & -0.65 & -1 & -0.98 & -0.36 \\
\hline 25 & bilateral & -0.04 & -0.02 & -0.18 & -0.52 & -0.35 & None & 1 & -0.55 \\
\hline 26 & right & -0.85 & None & -0.74 & 0.49 & None & -0.57 & -0.51 & -0.19 \\
\hline 27 & left & 0.24 & 0.42 & 0.90 & 0.97 & -0.10 & 0.22 & 0.33 & None \\
\hline 28 & left & 0.66 & 0.25 & 1 & -0.80 & None & 0.25 & 0.80 & 0.92 \\
\hline 29 & left & 0.14 & -0.38 & 0.21 & 0.69 & 0.81 & -0.92 & 0.49 & 0.37 \\
\hline 30 & left & 0.30 & -0.33 & -0.52 & 0.22 & -0.1 & -0.32 & 0.63 & 0.50 \\
\hline 31 & right & -0.89 & None & -1 & -1 & -1 & -0.38 & -1 & -0.81 \\
\hline 32 & left & 0.59 & 1 & 0.66 & 0.79 & 0.18 & None & 0.73 & None \\
\hline 33 & bilateral & -0.13 & 0.56 & -0.30 & -0.76 & -0.14 & 0.10 & 0.78 & -0.15 \\
\hline 34 & left & None & None & None & 1 & -0.94 & None & 0.59 & None \\
\hline 35 & left & 0.11 & 0.68 & 0.83 & 0.79 & -0.15 & 0.44 & -0.11 & None \\
\hline 36 & bilateral & -0.60 & -0.15 & 0.93 & -0.64 & -1 & -0.02 & None & 0.18 \\
\hline 37 & left & 0.71 & None & 0.55 & 0.40 & 0.34 & -0.42 & 0.35 & 0.53 \\
\hline 38 & left & 0.91 & 0.08 & 0.35 & None & 1 & -0.52 & 0.87 & 1 \\
\hline 39 & left & 0.54 & 0.15 & -0.97 & 0.35 & 0.45 & -0.14 & 0.64 & 0.29 \\
\hline 40 & right & -0.18 & -0.04 & None & -0.77 & None & 0.07 & -0.83 & -0.44 \\
\hline
\end{tabular}

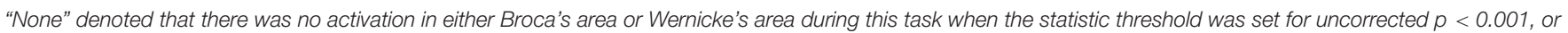

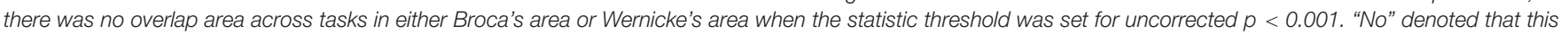
patient did not perform this language task.

is that the ESM result cannot support the right lateralization result. Because this patient was implanted electrodes on the left hemisphere, and resected on the left temporal lobe, there is no chance for us to verify the language function on the right hemisphere with ESM. On the other hand, the intact post-surgical language function maybe depended on the undetected left language related areas or the unverified right language related areas.

\section{Overlapping Activation Across Tasks}

Among the 40 patients enrolled in the study, 39 (97.5\%) showed overlapping activation across tasks $[29(72.5 \%)$ showed overlapping activations in both Broca's area and Wernicke's area; $10(25 \%)$ showed overlapping activations only in Broca's area]. Of the 39 patients who showed overlapping areas across tasks, 37 (94.9\%) showed that the LI of the overlapping areas was consistent with their language lateralization. 
TABLE 3 | Results of language testing.

\begin{tabular}{|c|c|c|c|}
\hline $\begin{array}{l}\text { Patients } \\
\text { no. }\end{array}$ & Cortical stimulation & $\begin{array}{l}\text { Language } \\
\text { lateralization }\end{array}$ & $\begin{array}{l}\text { Concordance } \\
\text { rating }\end{array}$ \\
\hline 8 & Positive on left temporal & Right & Low \\
\hline 12 & Positive on left frontal and temporal & Right & Moderate \\
\hline 13 & Positive on left temporal & Bilateral & High \\
\hline 14 & Negative on right frontal and temporal & Left & High \\
\hline 16 & Negative on right frontal and temporal & Left & High \\
\hline 17 & Negative on left frontal and temporal & Right & High \\
\hline 18 & Positive on left frontal and temporal & Right & Moderate \\
\hline 20 & Positive on left temporal & Right & Moderate \\
\hline 24 & Negative on left frontal and temporal & Right & High \\
\hline 25 & Positive on left frontal and temporal & Bilateral & High \\
\hline 26 & Negative on left frontal and temporal & Right & High \\
\hline 27 & Positive on left frontal and temporal & Left & High \\
\hline 28 & Positive on left frontal & Left & High \\
\hline 29 & Positive on left frontal and temporal & Left & High \\
\hline 30 & Positive on left frontal & Left & High \\
\hline 32 & Positive on left frontal & Left & High \\
\hline 33 & Positive on left frontal and temporal & Bilateral & High \\
\hline 35 & Positive on left frontal and temporal & Left & High \\
\hline 37 & Positive on left temporal & Left & High \\
\hline 38 & Positive on left temporal & Left & High \\
\hline
\end{tabular}

Of the 29 patients who performed the verb generation task, only one $(3.4 \%)$ showed no activation in either left or right language-related areas. In addition, 23 patients (79.3\%) showed that the LI of the verb generation task was consistent with language lateralization.

\section{Language Lateralization}

Among the 18 patients showing right-sided lateralization, 17 (94.4\%) had resection areas that involved the left Broca's area and Wernicke's area. The residual left language area detected by our tasks was remained. Furthermore, these patients all maintained an intact language function after surgery. Current data without ESM confirmed right-sided activation could hardly draw firm conclusion of right dominant language function. However, the fMRI activations across tasks on the right language areas and the intact post-operation language functions provided evidence for the indication of right-sided language lateralization. Of the 5 patients who suffered from multiple left ictal foci in the left hemisphere and underwent white matter disconnection surgery, they all showed rightsided lateralization of language processing. Their post-surgery language function remained intact. Figure 1 showed patient \#18's activation maps of five tasks (A, text reading; $B$, sentence reading; $\mathrm{C}$, word comprehension; $\mathrm{D}$, verb generation; and $\mathrm{E}$, auditory comprehension).

Of the 17 patients showing left-sided lateralization, 15 (88.2\%) had resection areas that involved the left Broca's area and Wernicke's area, and two (11.8\%) had resection areas that involved the right Broca's area and Wernicke's area. Three of these patients showed less fluency in post-surgical language function, whereas this function remained intact in the rest
14 patients. Figure 2 showed patient \#23's activation maps of five tasks ( $\mathrm{A}$, text reading; $\mathrm{B}$, sentence reading; $\mathrm{C}$, word comprehension; $\mathrm{D}$, verb generation; $\mathrm{E}$, visual object naming; and $\mathrm{F}$, auditory comprehension).

All five patients subjected to bilateral language processing had resection areas that involved the left Wernicke's area. One of these patients showed less fluency in post-surgical language function. Figure 3 showed patient \#33's activation maps of five tasks ( $\mathrm{A}$, text reading; $\mathrm{B}$, sentence reading; $\mathrm{C}$, word comprehension; $\mathrm{D}$, verb generation; $\mathrm{E}$, visual object naming; and $\mathrm{F}$, auditory comprehension).

\section{DISCUSSION}

In the present study, we evaluated the efficiency of our fMRI tasks in establishing hemispheric dominance for the Chinese language in patients with epilepsy. Localization of the language cortex and subsequent determination of laterality was successfully achieved in 40 patients. Our fMRI results showed favorable concordance with ESM in patients. We used the LI across various tasks and six individual tasks to measure the laterality for each patient. The relatively high rate of successful laterality assessments, as well as the selection of resection areas according to our assessment guaranteed intact post-surgical language function in patients with epilepsy, and make a strong case for the adoption of our fMRI tasks in Chinese language mapping as a suitable alternative to the Wada test and direct cortical stimulation mapping. The neurosurgeons in our department no longer apply the Wada test because of its invasiveness. Moreover, direct cortical stimulation is also invasive, and as it requires a bone window through which electrodes can be implanted, or an intraoperative wakeup test can be conducted, it may be difficult to perform from a practical perspective (in our study, only half of the patients were able to complete electrical stimulation mapping). Furthermore, direct cortical stimulation was only used for voluntary language assessment on one hemisphere. Our fMRI tasks provided a relatively comprehensive assessment of language-related areas, including both Broca's area and Wernicke's area. In order to detect more subtle activations for some of the less activated patients, the control tasks comprised either a blank screen or scanner noise.

The levels of our fMRI tasks, which included reading materials from spoken word to text, were thorough, and progressively investigated the ability to comprehend the Chinese language. The overlapping activation areas across tasks demonstrated the efficiency of the series of tasks among different patients. For clinical purposes, our fMRI tasks could provide more accurate identification of the language-related areas that should be protected during subsequent surgeries.

Our results of the verb generation task revealed that it could easily detect activations of the left hemisphere in language processing. However, it requires better cooperation and higher levels of education. Even in patients with rightsided language lateralization, residual left hemisphere activations were also important for the protection of language function. 

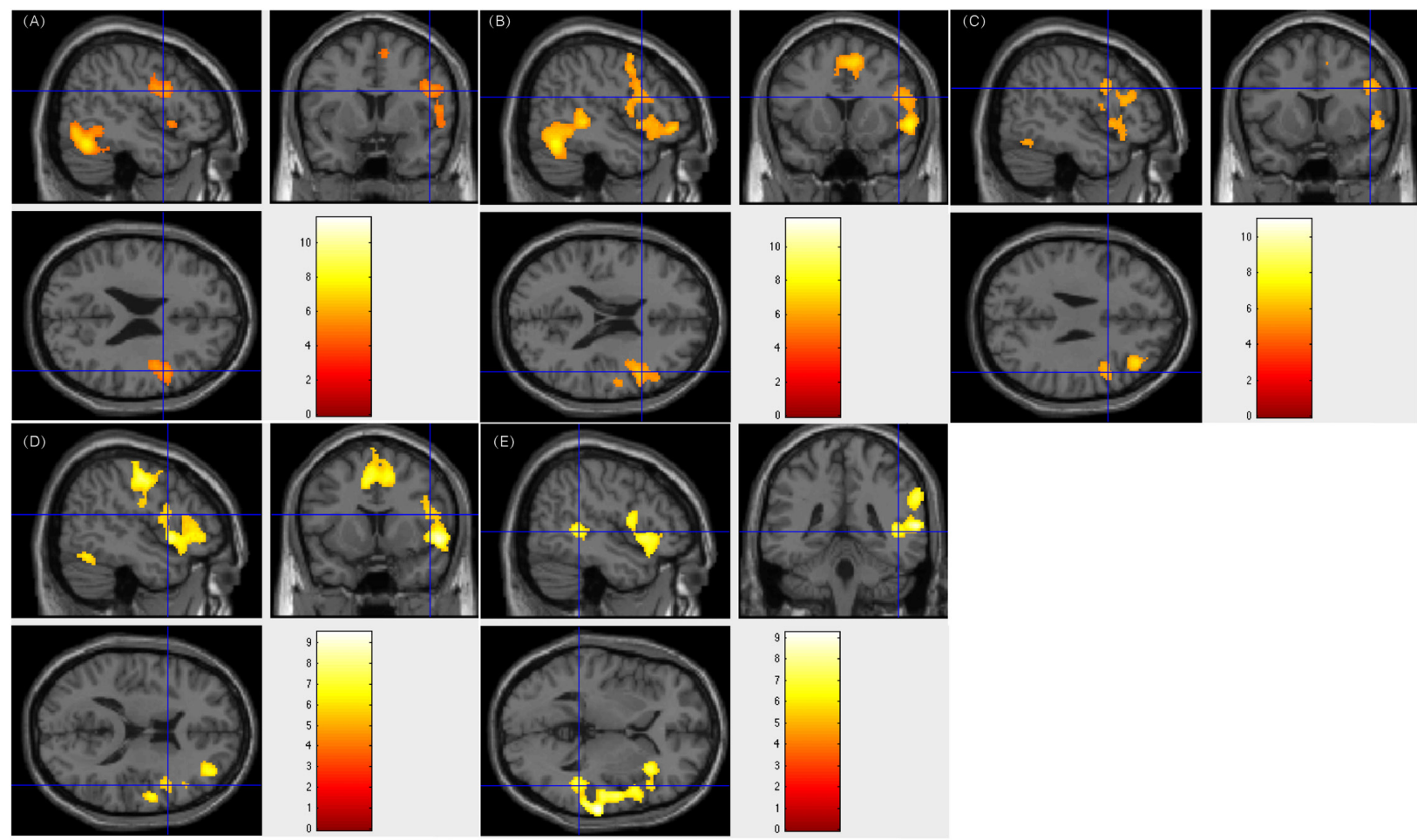

FIGURE 1 | Individual level activation of patient \#18 in language related areas showing contrast of task $>$ rest (fdr $p=0.001$ ). Different panel showed different task. (A) text reading; (B) sentence reading; (C) word comprehension; (D) verb generation; and (E) auditory comprehension.
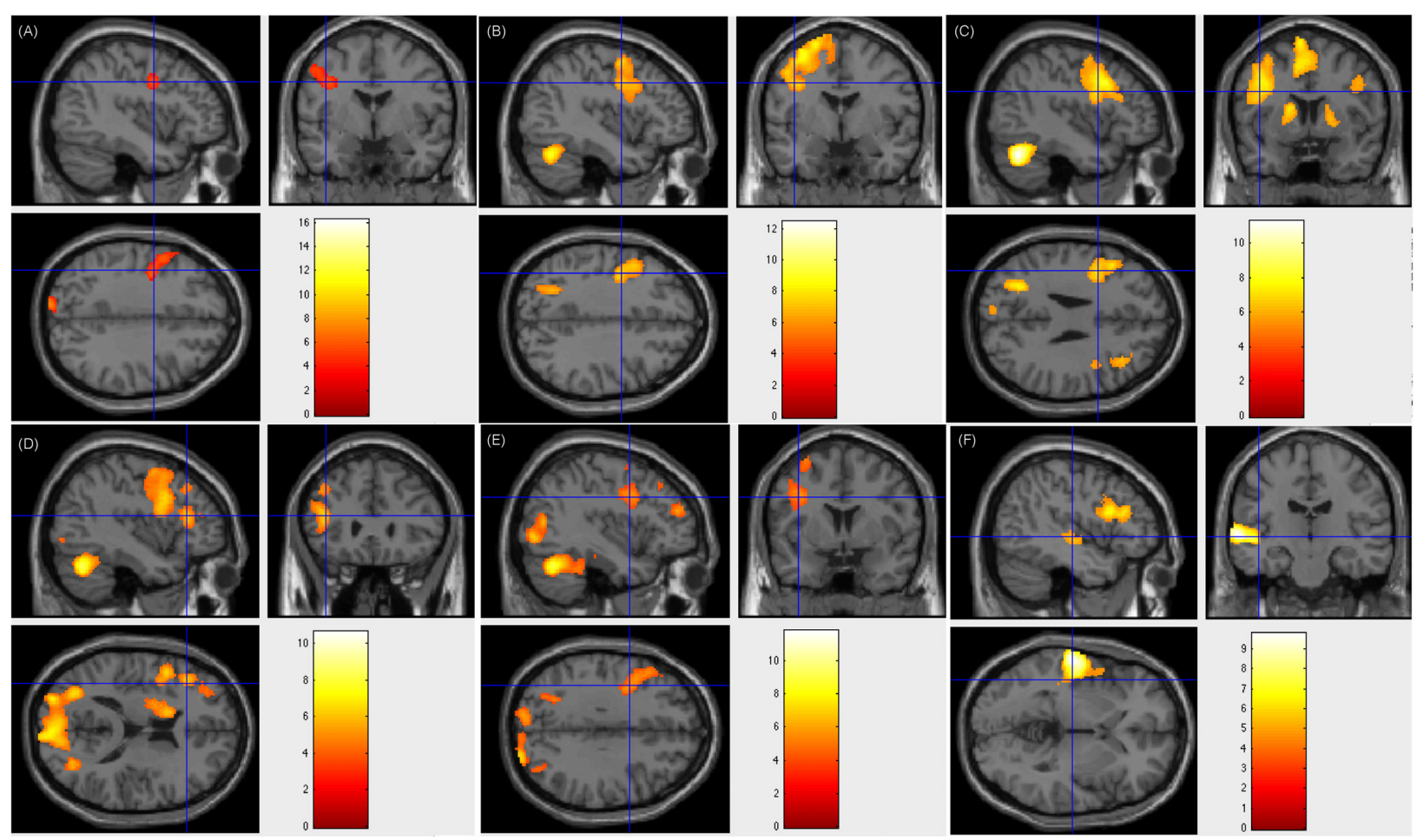

FIGURE 2 | Individual level activation of patient \#23 in language related areas showing contrast of task > rest (fdr $p=0.001)$. Different panel showed different task. (A) text reading; (B) sentence reading; (C) word comprehension; (D) verb generation; (E) visual object naming; and (F) auditory comprehension. 

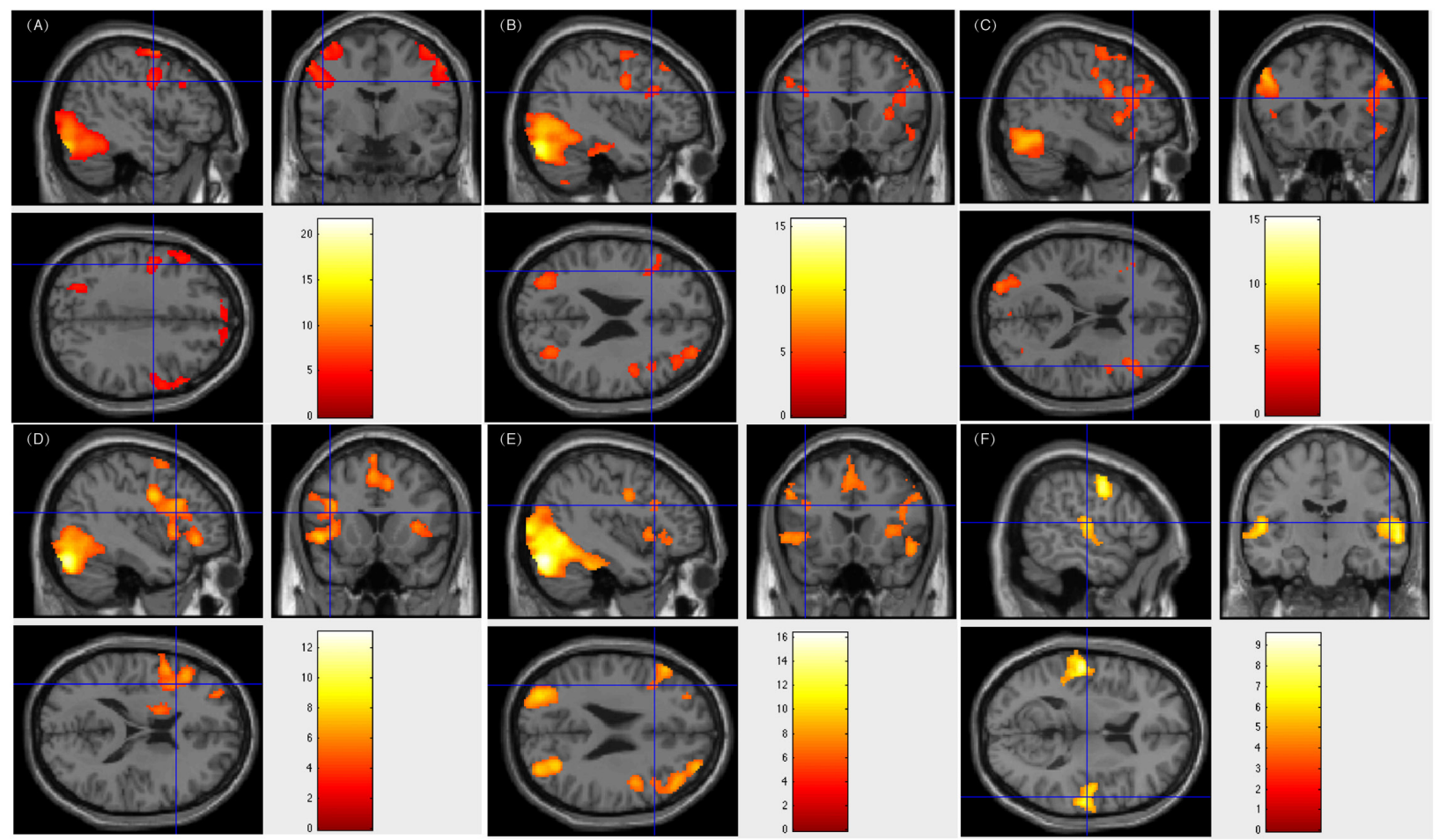

FIGURE 3 | Individual level activation of patient \#33 in language related areas showing contrast of task $>$ rest (fdr $p=0.001)$. Different panel showed different task. (A) text reading; (B) sentence reading; (C) word comprehension; (D) verb generation; (E) visual object naming; and (F) auditory comprehension.
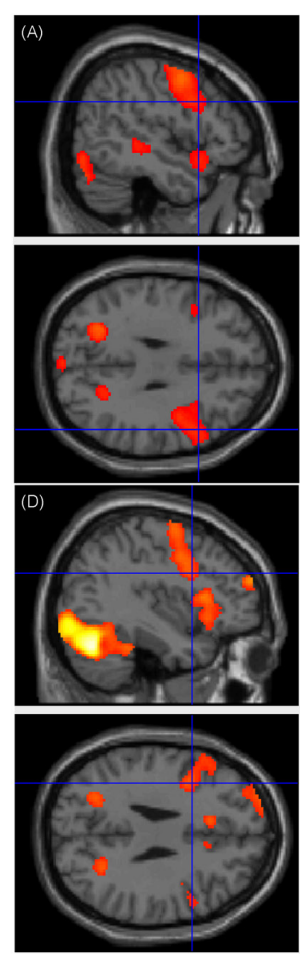
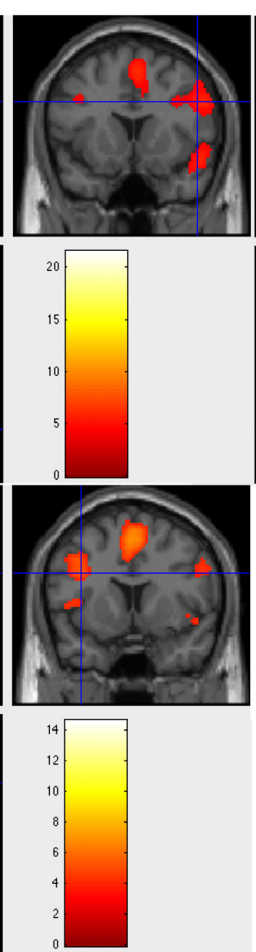
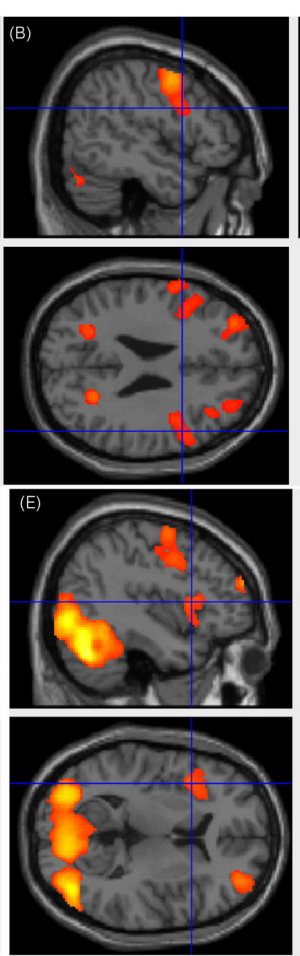
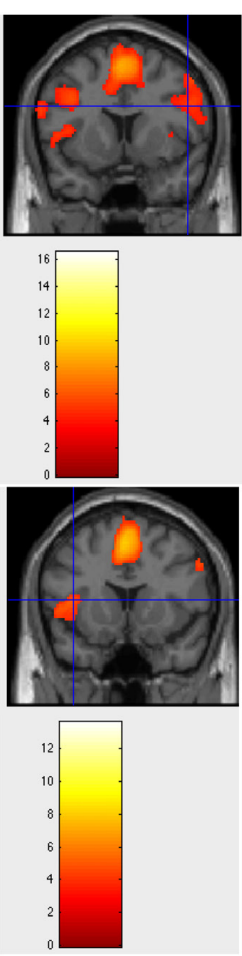
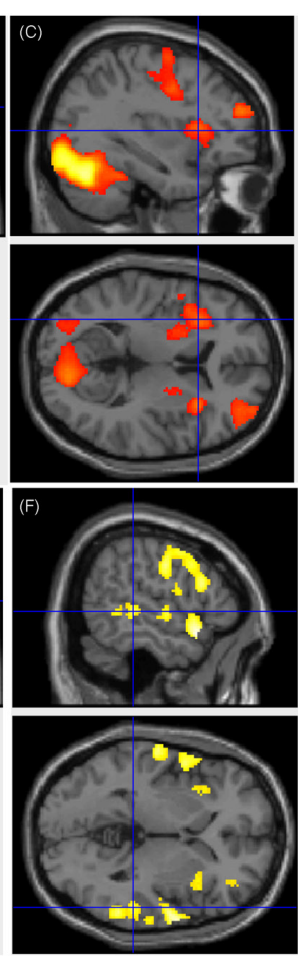
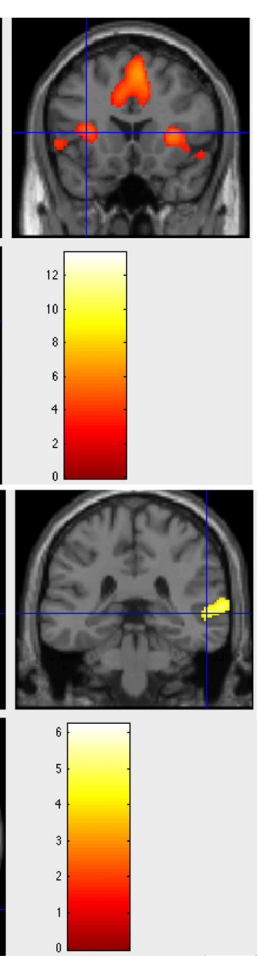

FIGURE 4 | Individual level activation of patient \#30 in language related areas showing contrast of task > rest (fdr $p=0.001)$. Different panel showed different task. (A) text reading; (B) sentence reading; (C) word comprehension; (D) verb generation; (E) visual object naming; and (F) auditory comprehension. 
Some patients with right-sided language lateralization were performed a white matter disconnection surgery. Limited by the clinical approach, they did not get a chance to verify their fMRI activations on the right language areas by ESM. Reliable fMRI activations on the right language areas helped the surgeons to make a resection plan on the left language areas, and their postsurgical language function remained intact. These results confirm the validity of our approach in assessing the lateralization of language processing.

Our fMRI tasks also corroborate and extend the finding that the left middle frontal area (BA 9) plays an important role in reading Chinese at multiple levels (text, sentence, word, and verb generation). Previous work (Tan et al., 2000) reported that compared to the fixation baseline, peak activations was elicited in the left middle frontal gyrus (BA 9) when the subjects were required to judge whether a pair of Chinese characters presented synchronously were related semantically. This finding was supported by other studies using similar Chinese word or character retrieval tasks (Tan, 2001a,b; Fu, 2002; Mo et al., 2005; Deng et al., 2015). Using our tasks regarding language comprehension and word retrieval (word generation task), which were different from those of Tan and colleagues, we could locate BA 9 in 39 (97.5\%) patients. In the patients with positive results of ESM on left frontal areas, left middle frontal area (BA 9) was verified. However, there were $2(5 \%)$ patients being activated only on left middle frontal area (BA 9), 30 (75\%) patients being activated on both left and right middle frontal area (BA 9), and 7 (17.5\%) patients being activated only on right middle frontal area (BA 9). Here we show Patient number 30's activation figures in Figure 4, whose resection area was left frontal area including part of BA 9 and ESM results was overlaid on left BA 9.

Collectively, our findings from multiple tasks in establishing hemispheric dominance for the Chinese language in patients with epilepsy are indicative of the efficiency of this series of fMRI tasks in facilitating surgical planning and the protection of postsurgical language function. Considering the challenges associated with assessing brain function in patients with epilepsy, who have various educational backgrounds, this study supports the utility of this method in pre-surgical language mapping.

The limitation in this method is the need of better design of fMRI tasks, including task control and the balance of scanning time and patient compliance. We intend to add more test blocks and replace the control condition on the premise of good completion. As for patients with various educational backgrounds, it is worth of investigation that whether to develop a method compatible for all the patients or subdivide the test for different groups of patients.

\section{REFERENCES}

Backes, W. H., Deblaere, K., Vonck, K., Kessels, A. G., Boon, P., Hofman, P., et al. (2005). Language activation distributions revealed by $\mathrm{fMRI}$ in post-operative epilepsy patients: Differences between left- and right-sided resections. Epilepsy Res. 66, 1-12. doi: 10.1016/j.eplepsyres.2005.06.007

Baxendale, S. (2009). The wada test. Curr. Opin. Neurol. 22:185. doi: 10.1097/WCO. 0b013e328328f32e
Future studies incorporating a multimodal-imaging framework may further highlight the efficiency of noninvasive methods of functional mapping in challenging populations with epilepsy.

\section{ETHICS STATEMENT}

This study was carried out in accordance with the recommendations of "The Institutional Review Boards of the Beijing MR Center for Brain Research" with written informed consent from all subjects. All subjects gave written informed consent in accordance with the Declaration of Helsinki. The protocol was approved by the "Institutional Review Boards of the Xuanwu Hospital.”

\section{AUTHOR CONTRIBUTIONS}

RW and BN contributed to designing the study and collecting the fMRI data. XW and TY contributed to conducting the ESM and other neurological examinations. RW and BW analyzed the data. $\mathrm{BN}$ and RW wrote the manuscript.

\section{FUNDING}

This work was supported in part by the Ministry of Science and Technology of China grant (2015CB351701), the National Natural Science Foundation of China grant (31730039), and the Strategic Priority Research Program of Chinese Academy of Sciences (XDB32010300).

\section{ACKNOWLEDGMENTS}

We would like to thank all the patients for their cooperation. Many thanks to Chang Liu, Xi Zhang, Xiaohua Zhang, and Xiaoming Yan. We also thank Kun Hu for her technique support.

\section{SUPPLEMENTARY MATERIAL}

The Supplementary Material for this article can be found online at: https://www.frontiersin.org/articles/10.3389/fnhum. 2019.00183/full\#supplementary-material

Billingsley, R. L., Mcandrews, M. P., Crawley, A. P., and Mikulis, D. J. (2001). Functional mri of phonological and semantic processing in temporal lobe epilepsy. Brain 124(Pt 6), 1218-1227. doi: 10.1093/brain/124.6.1218

Bookheimer, S. (2002). Functional mri of language: new approaches to understanding the cortical organization of semantic processing. Annu. Rev. Neurosci. 25:151. doi: 10.1146/annurev.neuro.25.112701.142946

Campo, P., Poch, C., Toledano, R., Igoa, J. M., Belinchón, M., García-Morales, I, et al. (2016). Visual object naming in patients with small lesions centered at 
the left temporopolar region. Brain Struct. Funct. 221, 473-485. doi: 10.1007/ s00429-014-0919-1

Carpentier, A., Pugh, K. R., Westerveld, M., Studholme, C., Skrinjar, O., Thompson, J. L., et al. (2010). Functional mri of language processing: dependence on input modality and temporal lobe epilepsy. Epilepsia 42, 1241 1254. doi: 10.1046/j.1528-1157.2001.35500.x

Cummine, J., Borowsky, R., Winder, F. S., and Crossley, M. (2009). Basic reading skills and dyslexia: three decades following right versus left hemispherectomy for childhood-onset intractable epilepsy. Epilepsy Behav. 15, 470-475. doi: 10. 1016/j.yebeh.2009.05.018

Deng, X., Zhang, Y., Xu, L., Wang, B., Wang, S., Wu, J., et al. (2015). Comparison of language cortex reorganization patterns between cerebral arteriovenous malformations and gliomas: a functional MRI study. J. Neurosurg. 122, 9961003. doi: 10.3171/2014.12.JNS14629

Duffau, H., Capelle, L., Denvil, D., Sichez, N., Gatignol, P., Lopes, M., et al (2003). Functional recovery after surgical resection of low grade gliomas in eloquent brain: hypothesis of brain compensation. J. Neurol. Neurosurg. Psychiatry 74, 901-907. doi: 10.1136/jnnp.74.7.901

Epstein, C. M. (1998). Transcranial magnetic stimulation: language function. J. Clin. Neurophysiol. 15, 325-332. doi: 10.1097/00004691-19980700000004

Fu, S. M., Chen, Y. P., Smith, S., Iversen, S., and Matthews, P. M. (2002). Effects of word form on brain processing of written Chinese. Neuroimage 17, 1538-1548. doi: 10.1006/nimg.2002.1155

Gaillard, W. D., Balsamo, L., Xu, B., Grandin, C. B., Braniecki, S. H., Papero P. H. et al. (2002). Language dominance in partial epilepsy patients identified with an fmri reading task. Neurology 59, 256-265. doi: 10.1212/wnl. 59.2.256

Hamberger, M. J. (2007). Cortical language mapping in epilepsy: a critical review. Neuropsychol. Rev. 17, 477-489. doi: 10.1007/s11065-007-9046-6

Kochunov, P., Fox, P., Lancaster, J., Tan, L. H., Amunts, K., Zilles, K., et al. (2003). Localized morphological brain differences between english-speaking caucasians and chinese-speaking asians: new evidence of anatomical plasticity. Neuroreport 14, 961-964. doi: 10.1097/00001756-200305230-00011

Matsuo, K., Chen, S. H. A., and Tseng, W. Y. I. (2012). AveLI: a robust lateralization index in functional magnetic resonance imaging using unbiased threshold-free computation. J. Neurosci. Methods 205, 119-129. doi: 10.1016/j.jneumeth.2011. 12.020

Mo, L., Liu, H. L., Jin, H., and Yang, Y. L. (2005). Brain activation during semantic judgment of Chinese sentences: a functional MRI study. Hum. Brain Mapp. 24, 305-312. doi: 10.1002/hbm.20091

Ojemann, G., Ojemann, J., Lettich, E., and Berger, M. (1989). Cortical language localization in left, dominant hemisphere. an electrical stimulation mapping investigation in 117 patients. J. Neurosurg. 71:316. doi: 10.3171/jns.1989.71. 3.0316

Petersen, S. E., Fox, P. T., Posner, M. I., Mintun, M., and Raichle, M. E. (1988). Positron emission tomographic studies of the cortical anatomy of single-word processing. Nature 331, 585-589. doi: 10.1038/331585a0
Qiu, J., Luo, Y., Wang, Q., Zhang, F., and Zhang, Q. (2006). Brain mechanism of stroop interference effect in Chinese characters. Brain Res. 1072, 186-193. doi: 10.1016/j.brainres.2005.12.029

Rodin, D., Bar-Yosef, O., Smith, M. L., Kerr, E., Morris, D., and Donner, E. J. (2013). Language dominance in children with epilepsy: concordance of fmri with intracarotid amytal testing and cortical stimulation. Epilepsy Behav. 29, 7-12. doi: 10.1016/j.yebeh.2013.06.005

Rutten, G. J., Ramsey, N. F., van Rijen, P. C., Alpherts, W. C., and van Veelen, C. W. (2002). Fmri-determined language lateralization in patients with unilateral or mixed language dominance according to the wada test. Neuroimage 17, 447-460. doi: 10.1006/nimg.2002.1196

Sabsevitz, D. S., Swanson, S. J., Hammeke, T. A., Spanaki, M. V., Possing, E. T., Rd, M. G., et al. (2003). Use of preoperative functional neuroimaging to predict language deficits from epilepsy surgery. Neurology 60, 1788-1792. doi: 10.1212/ 01.wnl.0000068022.05644.01

Strauss, E., and Wada, J. (1983). Lateral preferences and cerebral speech dominance. Cortex 19, 165-177. doi: 10.1016/s0010-9452(83)80012-4

Szaflarski, J. P., Binder, J. R., Possing, E. T., McKiernan, K. A., Ward, B. D., and Hammeke, T. A. (2002). Language lateralization in left-handed and ambidextrous people: fMRI data. Neurology 59, 238-244.? doi: 10.1212/wnl. 59.2 .238

Tan, L. H., Feng, C. M., Fox, P. T., and Gao, J. H. (2001a). An fMRI study with written Chinese. Neuroreport 12, 83-88. doi: 10.1097/00001756-20010122000024

Tan, L. H., Liu, H. L., Perfetti, C. A., Spinks, J. A., Fox, P. T., and Gao, J. H. (2001b). The neural system underlying Chinese logograph reading. Neuroimage 13, 836-846. doi: 10.1006/nimg.20 01.0749

Tan, L. H., Spinks, J. A., Gao, J. H., Liu, H. L., Perfetti, C. A., Xiong, J. H., et al. (2000). Brain activation in the processing of Chinese characters and words: a functional MRI study. Hum. Brain Mapp. 10, 16-27. doi: 10.1002/(sici)10970193(200005)10:1<16::aid-hbm30>3.0.co;2-m

Valaki, C. E., Maestu, F., Simos, P. G., Zhang, W., Fernandez, A., and Amo, C. M., et al (2004). Cortical organization for receptive language functions in chinese, english, and spanish: a cross-linguistic meg study. Neuropsychologia 42, 967-979. doi: 10.1016/j.neuropsychologia.2003. 11.019

Conflict of Interest Statement: The authors declare that the research was conducted in the absence of any commercial or financial relationships that could be construed as a potential conflict of interest.

Copyright (c) $2019 \mathrm{Ni}$, Wang, Yu, Wu and Wang. This is an open-access article distributed under the terms of the Creative Commons Attribution License (CC BY). The use, distribution or reproduction in other forums is permitted, provided the original author(s) and the copyright owner(s) are credited and that the original publication in this journal is cited, in accordance with accepted academic practice. No use, distribution or reproduction is permitted which does not comply with these terms. 